\title{
Making Planet Nine: A Scattered Giant in the Outer Solar System
}

\author{
Benjamin C. Bromley \\ Department of Physics 83 Astronomy, University of Utah, \\ 115 S 1400 E, Rm 201, Salt Lake City, UT 84112 \\ bromley@physics . utah.edu \\ Scott J. Kenyon \\ Smithsonian Astrophysical Observatory, \\ 60 Garden St., Cambridge, MA 02138 \\ skenyon@cfa.harvard.edu
}

\begin{abstract}
Correlations in the orbits of several minor planets in the outer solar system suggest the presence of a remote, massive Planet Nine. With at least ten times the mass of the Earth and a perihelion well beyond 100 AU, Planet Nine poses a challenge to planet formation theory. Here we expand on a scenario in which the planet formed closer to the Sun and was gravitationally scattered by Jupiter or Saturn onto a very eccentric orbit in an extended gaseous disk. Dynamical friction with the gas then allowed the planet to settle in the outer solar system. We explore this possibility with a set of numerical simulations. Depending on how the gas disk evolves, scattered super-Earths or small gas giants settle on a range of orbits, with perihelion distances as large as 300 AU. Massive disks that clear from the inside out on million-year time scales yield orbits that allow a super-Earth or gas giant to shepherd the minor planets as observed. A massive planet can achieve a similar orbit in a persistent, low-mass disk over the lifetime of the solar system.
\end{abstract}

Subject headings: Planetary systems - Planets and satellites: formation - planet disk interactions

\section{Introduction}

The orbital alignment of minor planets located well beyond Neptune, including Sedna and $2012 \mathrm{VP}_{113}$, inspired Trujillo \& Sheppard (2014) to invoke a massive, unseen planet orbiting at roughly $200 \mathrm{AU}$ from the Sun. Expanding on this analysis, Batygin \& Brown (2016; see also Brown \& Batygin 2016) propose a more distant planet which maintains the apsidal alignment for 
a set of six trans-Neptunian objects. With a mass more than ten times that of the Earth, this planet would have a semimajor axis between $300 \mathrm{AU}$ to $1500 \mathrm{AU}$, an eccentricity within the range of roughly $0.2-0.8$, an inclination below $40^{\circ}$, and an apsis that is anti-aligned with the six minor planets. Subsequent work by Fienga et al. (2016), using precise Cassini radio ranging data of Saturn, places constraints on the perturber's orbital phase.

The prospect of a Planet Nine lurking in the outer solar system provides a new opportunity to test our understanding of planet formation theory. Various mechanisms - coagulation (Kenyon \& Bromlev 2015), gravitational instability (Helled et al. 2014, and references therein), and scattering (Bromley \& Kenyon 2014) - can place a massive planet far from the host star. Aside from a direct detection of Planet Nine, testing these ideas requires numerical simulations which predict the properties of planets as a function of initial conditions in the protoplanetary disk.

Previous calculations of gas giant planet formation (Rasio \& Ford 1996; Weidenschilling \& Marzari 1996; Ford et al. 2005; Moorhead \& Adams 2005; Levison \& Morbidelli 2007; Chatterjee et al. 2008; Bromley \& Kenyon 2011) demonstrate that growing gas giants clear their orbital domains by scattering super-Earths or more massive planets to large distances. If the surface density of the gaseous disk at large distances is small, scattered planets are eventually ejected. For disks with larger surface densities, however, dynamical friction damps a scattered planet to lower eccentricity (Dokuchaev 1964; Rephaeli \& Salpeter 1980; Takeda 1988; Ostriker 1999; Kominami \& Ida 2002). Bromley \& Kenyon (2014) used simple models of disk-planet interactions to show that this mechanism plausibly circularizes the orbits of massive planets at 100-200 AU from the central star.

The Batygin \& Brown (2016) analysis poses a new challenge to scattering models. Although they propose a moderately eccentric orbit for their massive perturber, the planet has a semimajor axis beyond 300-400 AU. Our goal here is to show under what conditions a scattered planet can achieve this orbit through dynamical friction with a gas disk. We consider a wide range of planet and disk configurations, numerically simulate outcomes of these models, and assess how well they explain a Planet Nine in the outer solar system.

\section{Method}

To explore the possibility of a scattered origin for Planet Nine, we follow our earlier strategy (Bromley \& Kenyon 2014). We choose initial conditions for planetary orbits and gas disks and a mechanism for disk dissipation. We then track the orbital evolution with the $n$-body integration component of our Orchestra code (Bromley \& Kenyon 2006; Kenyon \& Bromley 2008; Bromley \& Kenvon 2011). In this section we provide details of the disk models and an overview of the numerical method, which includes an updated treatment of dynamical friction. 


\subsection{The disk models}

To set the stage for relocating a scattered planet from 5-15 AU into the outer solar system, we model the Sun's gas disk with the following prescription for surface density $\Sigma$, scale height $H$, and midplane mass density $\rho_{\text {gas }}$ :

$$
\begin{aligned}
& \Sigma(a, t)= \begin{cases}\Sigma_{0}\left(\frac{a}{a_{0}}\right)^{-1} e^{-t / \tau}, & \text { if } a_{\text {in }} \leq a \leq a_{\text {out }}, \\
0 & \text { otherwise, }\end{cases} \\
& H(a)=h_{0} a\left(\frac{a}{a_{0}}\right)^{2 / 7}, \\
& \rho_{\text {gas }}(a, t)=\frac{\Sigma(a, t)}{H(a)} .
\end{aligned}
$$

Here, $\Sigma_{0}$ sets the surface density at distance $a_{0} \equiv 1 \mathrm{AU}, a_{\text {in }}$ and $a_{\text {out }}$ are the inner and outer edges of the disk, and $h_{0}=0.05$ establishes the scale height of the flared disk (Kenvon \& Hartmann 1987; Chiang \& Goldreich 1997; Andrews \& Williams 2007; Andrews et al. 2009). The global surface density decay parameter $\tau=1-10$ Myr enables a homologous reduction in the surface density (Haisch et al. 2001). To allow the inner edge of the disk to expand, as in a transition disk, we adopt an expansion rate $\kappa_{\text {in }}$ :

$$
a_{\text {in }}(t)=a_{\text {in }}(0)+\kappa_{\text {in }} t,
$$

where the initial size of the inner cavity is $a_{\mathrm{in}}(0) \equiv 20$ AU. Observations of transition disks (Calvet et al. 2005; Currie et al. 2008; Andrews et al. 2011; Najita et al. 2015) suggest opening rates of $\mathrm{O}(10) \mathrm{AU} / \mathrm{Myr}$.

Toward estimating dynamical friction and gas drag, we assume that the sound speed in the gas is $c_{s} \approx H v_{\mathrm{Kep}} / a$, where $v_{\mathrm{Kep}}$ is the circular Keplerian speed at orbital distance $a$ from the Sun. We also assume that both $H$ and $c_{s}$ are independent of time. Armed with these variables we can determine the Mach number of a planet moving relative to the gas, and hence derive drag forces on the planet. These estimates include the effect of pressure support within the gas disk, which makes the bulk flow in the disk sub-Keplerian, with an orbital speed that is reduced from $v_{\mathrm{K}_{\text {or }}}$ by a factor of $\left(1-H^{2} / a^{2}\right)$ (e.g., Adachi et al. 1976; Weidenschilling 1977a; Youdin \& Kenvon 2013).

Table 1 lists disk model parameters. We distinguish two types of disks: static and evolving. Static disks have fixed surface density profiles and small total mass, $0.002 \mathrm{M}_{\odot}<M_{\text {disk }}<0.06 \mathrm{M}_{\odot}$ (2-60 $\mathrm{M}_{J}$, where $\mathrm{M}_{J}$ is the mass of Jupiter), which extend to 1600 AU. These models enable us to consider the possibility of long-term $(\gtrsim 100 \mathrm{Myr})$ planet-disk interactions. Evolving disks extend to $800 \mathrm{AU}$ with larger initial surface density and mass. In the most extreme case $\left(\Sigma_{n}=1000 \mathrm{~g} / \mathrm{cm}^{2}\right)$, the disk mass is half that of the Sun. Although improbable (cf. Andrews et al. 2013), this extreme disk mass allows us to explore the possibility of rapid, strong orbital damping. Because dynamical friction depends on the gas density, $\rho_{\text {gas }} \sim \Sigma / H$, we can scale results to less massive disks with smaller scale heights. 


\subsection{Scattered planets}

For each disk configuration in Table1, we carry out simulations with planets scattered to large ( $>1000 \mathrm{AU}$ ) distances. As summarized in Table 2, each planet is assigned a mass $m_{\bullet}$ and mean density $\rho_{\bullet}$, from which we infer a physical radius, $r_{\bullet}$. In our orbital dynamics code, the planet is launched from a perihelion distance of $r_{\text {peri }}=10 \mathrm{AU}$ with a speed that would take it to a specified aphelion distance $r_{\text {apo }}$ if it were on a Keplerian orbit about the Sun. We track the subsequent dynamical evolution with orbital elements calculated geometrically, since the disk potential can complicate the interpretation of osculating orbital elements. While the planet's orbit typically comes close to the nominal starting aphelion, it never makes it to $r_{\text {apo }}$ exactly due to dynamical friction with the gas and the disk's overall gravitational potential.

\subsection{Numerical approach}

To evolve planetary orbits in a gas disk, we follow Bromley \& Kenvon (2014). We use the orbit integrator in our hybrid $n$-body-coagulation code Orchestra to calculate the trajectory of individual planets around the Sun in the midplane of the disk. We calculate disk gravity using 2000 radial bins spanning the planet's orbit, assigning a mass to each bin according to Equation (1). We initially solve the Poisson equation by numerical integration, storing the results. The saved potential is updated as the disk evolves.

To estimate acceleration from dynamical friction, we adopt a parameterization similar to Lee \& Stahler (2014) in the absence of gas accretion (see also Dokuchaev 1964; Ruderman \& Spiegel 1971; Ostriker 1999):

$$
\frac{d \vec{v}}{d t}=-\frac{G^{2} \rho_{\text {gas }} m_{\bullet} \mu}{c_{s}^{2}} \frac{\left(1+4 \pi^{2} C_{\mathrm{df}}^{2} \mu^{2}\right)^{1 / 2}}{\left(1+\mu^{2}\right)^{2}} \frac{\vec{v}_{\mathrm{rel}}}{\left|\vec{v}_{\mathrm{rel}}\right|},
$$

where $\vec{v}_{\text {rel }}$ is the planet's velocity relative to the gas, $\mu \equiv\left|\vec{v}_{\text {rel }}\right| / c_{s}$ is the mach number, and $C_{\mathrm{df}}$ is a constant that depends on the geometry of the disk in the plane perpendicular to the planet's motion.

In evaluating the coefficient $C_{\text {df }}$, we previously only considered contributions from gas more distant than $H / 2$ from a planet (Bromley \& Kenyon 2014). Here we are less restrictive and include contributions from material closer to the planet. The drag acceleration thus has a piece from distant disk material in slab geometry (Bromley \& Kenyon 2014), along with a Coulomb logarithm (e.g., Binney \& Tremaine 2008):

$$
C_{\mathrm{df}} \approx 0.31+\ln \left[\max \left(1, \frac{H}{2 R}\right)\right]
$$

where the radius $R$ is

$$
R \equiv \max \left(r_{\bullet}, R_{s}\right)
$$


and

$$
R_{s}=\frac{1}{\mu_{x}^{2}-1} \frac{2 G m_{\bullet}}{c s^{2}} \quad\left[\mu_{x}^{2}=\max \left(\mu^{2}, 1.0001\right)\right]
$$

is an effective sonic radius (e.g., Thun et al. 2016).

With this formulation, our goal is to map how scattered planets with large eccentricities $(e \lesssim 1)$ damp to modest values $(e \sim 0.5)$ when the planet moves at supersonic speeds. Once the planet achieves low eccentricity, its subsequent evolution is complicated by differential torque exchange with the disk (Goldreich \& Tremaine 1980; Ward 1997) and accretion of disk material (Hoyle \& Lyttleton 1939; Lee \& Stahler 2011). We do not attempt to track this behavior. Our prescription underestimates dynamical friction in the subsonic and transonic regimes (cf. Ostriker 1999). Thus we follow a planet as its orbit damps, but stop the integration if it manages to fully circularize before the gas disappears.

\section{Results}

We ran over $10^{4}$ simulations to map out the parameter space of disk and planet configurations. To describe our results, we first consider low-mass, static disks, which isolate the physics of dynamical damping without the complications of disk evolution. To evaluate damping outcomes over the 1-10 Myr lifetimes of typical protoplanetary disks, we then consider a set of evolving disks.

\subsection{Relocation of a scattered planet in a long-lived disk}

In this set of simulations, we set up static disks with low surface density $\left(\Sigma_{0}=2-50 \mathrm{~g} / \mathrm{cm}^{2}\right)$, large radial extent $\left(a_{\text {out }}=1600 \mathrm{AU}\right)$, and big inner cavities $\left(a_{\text {in }}=50-200 \mathrm{AU}\right)$. We evolve scattered planets over a time $t=100$ Myr. Figure 1 shows several outcomes with planets of different masses. All planets follow the same track in $a-e$ space as they circularize; more massive planets evolve further along the track.

Figure 2 illustrates how evolution depends on the disk configuration. The plot shows three separate evolutionary tracks in $a-e$ space, each corresponding to a different inner edge of the disk

$\left(a_{\text {in }}\right)$. With a smaller inner edge, the disk causes a planet to settle more quickly (because there is more disk material to interact with) and closer to the Sun. The markers in the plot designate how far each planet evolves. Planets in low surface density disks make less progress along their track than those in high surface density disks.

These calculations establish an approximate degeneracy between mass and the surface density parameter $\Sigma_{0}$ in the formula for dynamical friction acceleration (Equation (50)). As a result, the progress that a planet makes along its $a-e$ track in a fixed amount of time depends only on the product, $m_{\bullet} \times \Sigma_{0}$. Thus, data points showing the orbital evolution as a function of planet mass in Figure 1 can also represent the progress of a planet of fixed mass in disks with different surface 
densities. Similarly points in Figure 2 can represent outcomes with different planet masses at fixed surface density.

If long-lived disks are responsible for settling a planet on the type of orbit inferred by Batygin \& Brown (2016), then our suite of simulations suggests the following condition leads to successful Planet Ninelike outcomes:

$$
\left(\frac{\Sigma_{0}}{10 \mathrm{~g} / \mathrm{cm}^{2}}\right)\left(\frac{m_{\bullet}}{20 \mathrm{M}_{\oplus}}\right) \approx\left(\frac{a_{\mathrm{in}}}{100 \mathrm{AU}}\right)^{1 / 2}\left(\frac{t}{100 \mathrm{Myr}}\right)^{-1} .
$$

This expression applies as long as $a_{\text {out }} \gg a_{\text {in }}$. While it is only approximate, this relation suggests that a persistent, low-mass disk $\left(\Sigma_{0} \approx 0.3 \mathrm{~g} / \mathrm{cm}^{2}\right.$; about a quarter of a Jupiter mass in gas $)$ can modestly damp a scattered Neptune-size body within the age of the solar system.

\subsection{Settling in an evolving disk}

Observations indicate that the youngest stars are surrounded with opaque disks of gas and dust (see Kenvon \& Hartmann 1995; Kenvon et al. 2008; Williams \& Cieza 2011; Andrews 2015). Surface densities vary; the "Minimum Mass Solar Nebula" value of $\Sigma_{0} \approx 2000 \mathrm{~g} / \mathrm{cm}^{2}$ (Weidenschilling 1977b; Havashi 1981) is at the upper end of the range observed in the youngest stars (e.g., Andrews et al. 2013; Najita \& Kenvon 2014). These disks globally dissipate on a time scale, $\tau$, of millions of years (Haisch et al. 2001), and may also erode from the inside out at a rate of $\kappa_{\text {in }} \gtrsim O(10) \mathrm{AU} / \mathrm{Myr}$, as in transition disks (e.g., Andrews et al. 2011; Najita et al. 2015). The resulting behavior of a scattered planet as it settles depends sensitively on how mass is distributed in these disks as they evolve.

Figure 3 illustrates the dependence of planetary settling on disk parameters $\tau, \kappa_{\text {in }}$, the initial disk surface density, and the inner disk edge. Adopting a baseline model where $\left(\Sigma_{0}, a_{\text {in }}, \kappa_{\text {in }}, \tau\right)=$ (1000 g/ $\left.\mathrm{cm}^{2}, 60 \mathrm{AU}, 40 \mathrm{AU} / \mathrm{Myr}, 4 \mathrm{Myr}\right)$, we vary individual disk parameters for planets with masses of $15-30 \mathrm{M}_{\oplus}$, scattered to starting distances of $r_{\text {apo }}=2000-2800$ AU. The general trends are clear. Dynamical settling to small orbital distance and low eccentricity is more effective in massive, slowly evolving disks with small inner cavities.

Figure 4 summarizes the outcomes of all of our evolving disk models (see Tables 1 and 21). The trends that emerged in Figure 3 are apparent in this Figure as well: long-lived, massive disks lead to significant dynamical evolution, while short-lived, low-mass disks do not. Figure 4 also shows an extended "sweet spot" in $a-e$ space, labeled with "Planet Nine," roughly corresponding to orbital elements of the massive perturber hypothesized by Batygin \& Brown (2016) and Brown \& Batygin (2016). Several hundred models yield planets that lie in the sweet spot, suggesting that the scattering mechanism can explain the inferred orbit of Planet Nine in the outer solar system.

Despite the trends revealed in Figures 1 3, it is difficult to tell which set of model parameters leads to successful Planet Nine-like orbits. To distinguish models in a way that highlights successful 
ones, we define two variables,

$$
\begin{aligned}
P & \equiv\left(\frac{m \bullet}{10 \mathrm{M}_{\oplus}}\right)\left(\frac{\Sigma_{0}}{1000 \mathrm{~g} / \mathrm{cm}^{2}}\right)\left(\frac{r_{\text {apo }}}{1000 \mathrm{AU}}\right)^{-1} \\
Q & \equiv\left(\frac{\tau}{1 \mathrm{Myr}}\right)\left[\left(\frac{\kappa_{\text {in }}}{60 \mathrm{AU} / \mathrm{Myr}}\right)\left(\frac{a_{\text {in }}}{20 \mathrm{AU}}\right)\left(\frac{r_{\text {apo }}}{1000 \mathrm{AU}}\right)\right]^{-1} .
\end{aligned}
$$

Roughly, the first variable is a mass-dependent damping rate, determined by planet mass and the disk mass, along with a factor of $1 / r_{\text {apo }}$ that reduces this rate if the planet is launched further away from the Sun. The second one measures the disk lifetime, based on the global disk decay time and the time for the disk to clear from the inside out, along with geometric factors involving the disk's radial extent and the planet's initial orbit. For models where $\tau$ is formally infinite, we set $\tau=10 \mathrm{Myr}$, the simulated duration of the evolving disk models.

The variables $P$ and $Q$ help to isolate the parameters that are necessary for a scattered giant planet to settle on a Planet Nine-like orbit. Our choice for defining these quantities, along with the mass, length and time scales in Equations (10) and (11), are based more on simplicity than anything else; other combinations of model parameters may serve the same purpose. Nonetheless, our choice yields a nicely compact region in $P-Q$ space for models that succeed in matching Batygin and Brown's (2016) criteria for Planet Nine.

Figure 5] shows a swath of points in the $P-Q$ plane that correspond to successful models. These points have just the right balance between the masses of the disk and the planet $(P)$ on the one hand, and disk lifetime $(Q)$ on the other. Models without this balance tend to produce planets that circularize at small semimajor axes (high $P$ and high $Q$; large masses and long disk lifetimes) or remain highly eccentric at large semimajor axes (low $P$ and low $Q$; small masses and short disk lifetimes).

A rough quantitative relationship between $P$ and $Q$ for successful models is $Q \sim P^{-3 / 2}$; in terms of model parameters, this condition translates to:

$$
\left(\frac{m_{\bullet}}{1 \mathrm{M}_{\oplus}}\right)\left(\frac{\Sigma_{0}}{1000 \mathrm{~g} / \mathrm{cm}^{2}}\right) \approx\left(\frac{\kappa_{\text {in }}}{40 \mathrm{AU} / \mathrm{Myr}}\right)^{2 / 3}\left(\frac{a_{\mathrm{in}}}{40 \mathrm{AU}}\right)^{2 / 3}\left(\frac{\tau}{1 \mathrm{Myr}}\right)^{-2 / 3}\left(\frac{r_{\text {apo }}}{1000 \mathrm{AU}}\right)^{-1 / 3}
$$

from which the inverse relationship between disk lifetime and mass factors is apparent, as is the sensitivity to disk and orbit geometries.

\subsection{Summary of simulation outcomes}

These simulations suggest a broad range of outcomes in $a-e$ space for $1-50 \mathrm{M}_{\oplus}$ planets scattered from $10 \mathrm{AU}$ into the outer part of a gaseous disk. For many combinations of input parameters, planets remain on $e \gtrsim 0.80$ orbits at $a \gtrsim 400 \mathrm{AU}$. Another set of parameters yields in massive planets on nearly circular orbits at 100-200 AU from the host star. Specific combinations of disk 
and planet properties result in "successful models," with planets on orbits consistent with the massive perturber of Batygin \& Brown (2016):

1. A planet scattered at low inclination into a low-mass, long-lived disk damps at a rate proportional to the planet mass and the disk's surface density. The final semimajor axis depends on $a_{\text {in }}$, the inner radius of the disk; successful models have $a_{\text {in }} \gtrsim 50$ AU. A power-law disk $(\Sigma \sim 1 / a)$ with low surface density $\left(\Sigma \sim 3 \times 10^{-3} \mathrm{~g} / \mathrm{cm}^{2}\right.$ at $100 \mathrm{AU}$, extending to $\left.\sim 1000 \mathrm{AU}\right)$ can produce a Neptune-size Planet Nine on a moderately eccentric orbit within the lifetime of the solar system. Higher planet masses or larger surface densities lead to success in less time.

2. Scattering in a massive, short-lived disk leads to Planet Nine-like orbits when there is a balance between the damping rate and the disk evolution time scale. In successful models, planet masses are typically $10 \mathrm{M}_{\oplus}$ or more, although $5 \mathrm{M}_{\oplus}$ planets can acquire a Planet Ninelike orbit in the most massive, long-lived disks. Most successful models experience either slow global decay $(\tau=4 \mathrm{Myr})$ or none at all. The disk then evolves primarily through inside-out erosion, as in a transition disk. This feature helps successful planets settle at large semimajor axes.

3. In all of our simulations, successful models tend to have semimajor axes that lie within $a=$ $600 \mathrm{AU}$. Batygin and Brown's preferred model has a higher orbital distance, with $a \approx 700 \mathrm{AU}$ (see also Malhotra et al. 2016; Brown \& Batygin 2016). While their analysis accommodates a wide range of possibilities, our current models do not. If a massive perturber were to have a semimajor axis firmly established beyond 700 AU, our mechanism would require a disk with more mass beyond a few hundred AU.

\section{Discussion}

Although a Planet Nine in the outer solar system has not yet been confirmed, several massive exoplanets have been identified at large distances from their host stars. The outermost planet in the HR 8799 system has a semimajor axis of $a \approx 70$ AU (Marois et al. 2008; Maire et al. 2015). The planets in 1RXS J160929.1-210524 ( $a \approx 330$ AU; Lafrenière et al. 2010), and HD $106906 \mathrm{~b}$ $(a \approx 650$ AU Bailey et al. 2014) have much larger semimajor axes. Gravitational instability is a popular mechanism to produce planets with such large $a$ (e.g., Helled et al. 2014; Rice 2016). Our calculations demonstrate that a planet scattered from $a \approx 10$ AU can interact with a gaseous disk and settle on roughly circular orbits at much larger $a$. Thus, scattering is a viable alternative to disk instability for placing massive planets at large $a$.

In our approach, we do not consider whether a scattered planet might accrete gas as its orbit damps (cf. Hoyle \& Lyttleton 1939). In principle, planets at large a might accumulate significant amounts of gas in 1-10 Myr. Whether accreting planets end up in configurations similar to those 
of the gas giants in HR 8799, 1RXS J160929.1-210524, and HD 106906 b requires an expanded set of more physically realistic simulations which are beyond the scope of the present work.

Here, we have focused on identifying initial conditions that yield a planet of fixed mass on an orbit with $e \sim 0.2-0.8$ at $a \gtrsim 300$ AU. With over $10^{4}$ models, we survey a variety of planet masses, scattered orbits, and configurations of the gas disk. A large central cavity in the disk, as observed in some transition disks (e.g., Andrews et al. 2011), is essential to settling Planet Nine at large orbital distances. Throughout, we assume that scattering and subsequent damping occur at low inclination; this condition is necessary for optimal interaction with the disk. A low scattering inclination is also expected. Damping by gas and planetesimals in the gas giant region likely kept larger bodies on orbits that were nearly coplanar with the gas disk (e.g., Lissauer \& Stewart 1993).

Our "successful" models - with outcomes that have the orbital characteristics of Batygin and Brown's (2016) inferred massive perturber - are those that balance planet and disk masses with disk longevity. In models where the disk is long-lived but low-mass, a planet like Neptune can settle within a few billion years. Successful models with more rapid gas dissipation require more massive disks. Disks that evolve on time scales of a few million years can lead to Planet Nine-like orbits, only if the initial disk mass is about $0.1 \mathrm{M}_{\odot}$ or more. A smaller disk scale height and/or reduced flaring of the disk (e.g. Keane et al. 2014) can reduce this restriction on the disk mass.

In addition to our proposal for scattering and damping as an origin for a massive perturber in the outer solar system, there are other compelling possibilities. These include in situ formation, late-time dynamical instabilities (the Nice model), passing stars, and Galactic tides. Each of these phenomena lead to different outcomes for Planet Nine.

In situ formation of Planet Nine is possible when disk evolution produces a massive ring of solids beyond 100 AU. Coagulation may then grow super-Earths in 1-5 Gyr out to distances of 750 AU (Kenyon \& Bromley 2015, 2016). In this mechanism, super-Earths reside on fairly circular orbits. For comparison, scattered planets can damp to circular orbits only inside of $\sim 200$ AU (see Fig. 4). Thus, a Planet Nine found at a large orbital distance with low eccentricity and low inclination strongly favors in situ formation. While not the favored choice of Batygin \& Brown (2016), it is unclear whether current observations explicitly rule out circular orbits for the massive perturber.

Other purely dynamical events can also produce a Planet Nine. In the Nice model (Tsiganis et al. 2005), a dynamical instability after the gaseous disk has dispersed can scatter a fully-formed giant planet into the outer solar system. Most scattered planets are ejected (e.g., Nesvorný 2011). If damping within a residual, low surface density gaseous disk is possible, some scattered planets might be retained on high eccentricity $(e \gtrsim 0.9)$, low inclination $\left(i \lesssim 10^{\circ}\right)$ orbits (e.g., Marzari et al. 2010; Ravmond et al. 2010). For a massive Planet Nine, the high $e$ orbit might distinguish this mechanism from our model, where scattering occurs when the inner edge of the disk lies much closer to the Sun.

A passing star — perhaps a member of the Sun's birth cluster (Adams \& Laughlin 2001) - 
can also relocate Planet Nine in the outer solar system. Outcomes vary widely, depending on the planet's initial orbit (e.g. Kobavashi \& Ida 2001; Kenyon \& Bromley 2004; Morbidelli \& Levison 2004; Brasser et al. 2006; Kaib \& Quinn 2008). If the planet starts on a circular orbit in the ecliptic plane, a stellar flyby will give it a strong kick in eccentricity but only a mild boost in perihelion distance and inclination. Thus, if the planet's present-day semimajor axis is above $400 \mathrm{AU}$, it is likely to have an eccentricity of 0.9 or more, unless it formed well beyond Neptune.

A passing star can yield a broader range of outcomes if Planet Nine were on an eccentric orbit at the time of the flyby, perhaps as a result of a previous stellar encounter or the scattering mechanism considered here. Alternatively, if the Sun captured Planet Nine from the passing star, the possibilities are even greater (e.g., Figures 2 and 3 of Kenyon \& Bromley 2004, see also Morbidelli \& Levison 2004, Levison et al. 2010, Jílková et al. 2015). However, the likelihood of this eventuality seems low (Li \& Adams 2016).

Finally, we consider the effect of tides from the Galactic environment. For Oort cloud comets, the gravitational potential of the Galaxy dominates the orbital evolution (Heisler \& Tremaine 1986; Duncan et al. 1987). However, tidal effects become weak inside $10^{4} \mathrm{AU}$; evolutionary time scales are then long, $100 \mathrm{Myr}$ or more. For objects with a semimajor axis within $1000 \mathrm{AU}$, the Galactic tide causes only small changes in the orbit over the age of the solar system (Higuchi et al. 2007; Brasser et al. 2008). In our static disk models, the semimajor axes we consider are at $800 \mathrm{AU}$ and smaller; a putative Planet Nine is then shielded from tidal interaction. In the evolving disk models, we use a maximum semimajor axis of 1800 AU. However, in successful models, the semimajor axis falls well below $700 \mathrm{AU}$ within $10 \mathrm{Myr}$, well within the tidal evolution time scale.

In the absence of any gas, the Galactic tide can influence the orbit of a Planet Nine initially scattered beyond $\sim 1000$ AU within a billion years of the solar system's formation. Torque from the Galactic potential then raises both the perihelion and the inclination of the orbit (e.g., Duncan et al. 1987; Higuchi et al. 2007; Brasser et al. 2008). The hallmark of this process would be a semimajor axis exceeding $1000 \mathrm{AU}$, a perihelion distance of at least $100 \mathrm{AU}$, an eccentricity of 0.8-0.9, and an inclination that may be anywhere from $i=0^{\circ}$ to $\sim 135^{\circ}$ (e.g., Figures 9 and 11 of Higuchi et al. 2007).

Tides from the Sun's birth cluster may have had an even more dramatic effect than the Galactic tide (e.g., Brasser et al. 2006). However, if this cluster was typical of other embedded clusters, it would have disintegrated quickly, within 2-3 Myr (see Lada \& Lada 2003). The density of stars in the cluster, the Sun's orbit through it, and the timing of the cluster dispersal relative to the formation of the gas giants are all uncertain. If Planet Nine's final orbit was determined by interactions during this phase of the Sun's history, then its high perihelion distance would also likely be accompanied by a high inclination (e.g., Figures 6 and 8 of Brasser et al. 2006).

Observations of exoplanetary systems provide ways to test these scenarios. Over the next 10$20 \mathrm{yr}$, direct imaging will probably yield large samples of gas giants at large $a$. Comparison of the observed properties of these systems with the predictions of numerical simulations should enable 
constraints on the likelihood of any particular theoretical model. For stars with ages of 5-10 Myr, current data suggest many systems with $\lesssim 1$ Jupiter mass of gas (e.g., Dent et al. 2013). Expanding surveys to older stars and reducing upper limits on the mass in gas by an order of magnitude would challenge some of our scattering models.

In the solar system, identifying Planet Nine and new dwarf planets is essential for making progress. As outlined in Batygin \& Brown (2016), larger samples of dwarf planets provide additional constraints on any Planet Nine. A robust detection of a massive perturber (see Cowan et al. 2016; Linder \& Mordasini 2016; Ginzburg et al. 2016; de la Fuente Marcos \& de la Fuente Marcos 2016) and direct measurement of orbital elements allow discrimination between the various possibilities for the origin and evolution of Planet Nine. If interactions with a gas disk turn out to be important, the next step is to obtain more realistic predictions of scattering outcomes with hydro-

dynamical simulations. Combined with observations of exoplanets, these advances might determine the fate of scattered planets.

We are grateful to M. Geller, J. Najita and D. Wilner for comments and helpful discussions. NASA provided essential support for this program through a generous allotment of computer time on the NCCS 'discover' cluster and Outer Planets Program grant NNX11AM37G.

\section{REFERENCES}

Adachi, I., Hayashi, C., \& Nakazawa, K. 1976, Progress of Theoretical Physics, 56, 1756

Adams, F. C., \& Laughlin, G. 2001, Icarus, 150, 151

Andrews, S. M. 2015, PASP, 127, 961

Andrews, S. M., Rosenfeld, K. A., Kraus, A. L., \& Wilner, D. J. 2013, ApJ, 771, 129

Andrews, S. M., \& Williams, J. P. 2007, ApJ, 671, 1800

Andrews, S. M., Wilner, D. J., Espaillat, C., et al. 2011, ApJ, 732, 42

Andrews, S. M., Wilner, D. J., Hughes, A. M., Qi, C., \& Dullemond, C. P. 2009, ApJ, 700, 1502

Bailey, V., Meshkat, T., Reiter, M., et al. 2014, ApJ, 780, L4

Batygin, K., \& Brown, M. E. 2016, AJ, 151, 22

Binney, J., \& Tremaine, S. 2008, Galactic Dynamics: Second Edition (Princeton University Press)

Brasser, R., Duncan, M. J., \& Levison, H. F. 2006, Icarus, 184, 59

—. 2008, Icarus, 196, 274 
Bromley, B. C., \& Kenyon, S. J. 2006, AJ, 131, 2737

-. 2011, ApJ, 731, 101

—. 2014, ApJ, 796, 141

Brown, M. E., \& Batygin, K. 2016, ArXiv e-prints, arXiv:1603.05712

Calvet, N., D’Alessio, P., Watson, D. M., et al. 2005, ApJ, 630, L185

Chatterjee, S., Ford, E. B., Matsumura, S., \& Rasio, F. A. 2008, ApJ, 686, 580

Chiang, E. I., \& Goldreich, P. 1997, ApJ, 490, 368

Cowan, N. B., Holder, G., \& Kaib, N. A. 2016, ArXiv e-prints, arXiv:1602.05963

Currie, T., Kenyon, S. J., Balog, Z., et al. 2008, ApJ, 672, 558

de la Fuente Marcos, C., \& de la Fuente Marcos, R. 2016, ArXiv e-prints, arXiv:1603.06520

Dent, W. R. F., Thi, W. F., Kamp, I., et al. 2013, PASP, 125, 477

Dokuchaev, V. P. 1964, Soviet Ast., 8, 23

Duncan, M., Quinn, T., \& Tremaine, S. 1987, AJ, 94, 1330

Fienga, A., Laskar, J., Manche, H., \& Gastineau, M. 2016, ArXiv e-prints, arXiv:1602.06116

Ford, E. B., Lystad, V., \& Rasio, F. A. 2005, Nature, 434, 873

Ginzburg, S., Sari, R., \& Loeb, A. 2016, ArXiv e-prints, arXiv:1603.02876

Goldreich, P., \& Tremaine, S. 1980, ApJ, 241, 425

Haisch, Jr., K. E., Lada, E. A., \& Lada, C. J. 2001, ApJ, 553, L153

Hayashi, C. 1981, Progress of Theoretical Physics Supplement, 70, 35

Heisler, J., \& Tremaine, S. 1986, Icarus, 65, 13

Helled, R., Bodenheimer, P., Podolak, M., et al. 2014, Protostars and Planets VI, 643

Higuchi, A., Kokubo, E., Kinoshita, H., \& Mukai, T. 2007, AJ, 134, 1693

Hoyle, F., \& Lyttleton, R. A. 1939, Proceedings of the Cambridge Philosophical Society, 35, 405

Jílková, L., Portegies Zwart, S., Pijloo, T., \& Hammer, M. 2015, MNRAS, 453, 3157

Kaib, N. A., \& Quinn, T. 2008, Icarus, 197, 221

Keane, J. T., Pascucci, I., Espaillat, C., et al. 2014, ApJ, 787, 153 
Kenyon, S. J., \& Bromley, B. C. 2004, Nature, 432, 598

-. 2008, ApJS, 179, 451

-. 2015, ApJ, 806, 42

—. 2016, ApJ, submitted (available on the arXiv)

Kenyon, S. J., Gómez, M., \& Whitney, B. A. 2008, in Handbook of Star Forming Regions, Volume I, ed. Reipurth, B. (San Francisco: Astronomical Society of the Pacific), 405-+

Kenyon, S. J., \& Hartmann, L. 1987, ApJ, 323, 714

—. 1995, ApJS, 101, 117

Kobayashi, H., \& Ida, S. 2001, Icarus, 153, 416

Kominami, J., \& Ida, S. 2002, Icarus, 157, 43

Lada, C. J., \& Lada, E. A. 2003, ARA\&A, 41, 57

Lafrenière, D., Jayawardhana, R., \& van Kerkwijk, M. H. 2010, ApJ, 719, 497

Lee, A. T., \& Stahler, S. W. 2011, MNRAS, 416, 3177

-. 2014, A\&A, 561, A84

Levison, H. F., Duncan, M. J., Brasser, R., \& Kaufmann, D. E. 2010, Science, 329, 187

Levison, H. F., \& Morbidelli, A. 2007, Icarus, 189, 196

Li, G., \& Adams, F. C. 2016, ArXiv e-prints, arXiv:1602.08496

Linder, E. F., \& Mordasini, C. 2016, ArXiv e-prints, arXiv:1602.07465

Lissauer, J. J., \& Stewart, G. R. 1993, in Protostars and Planets III, ed. E. H. Levy \& J. I. Lunine, $1061-1088$

Maire, A.-L., Skemer, A. J., Hinz, P. M., et al. 2015, A\&A, 576, A133

Malhotra, R., Volk, K., \& Wang, X. 2016, ArXiv e-prints, arXiv:1603.02196

Marois, C., Macintosh, B., Barman, T., et al. 2008, Science, 322, 1348

Marzari, F., Baruteau, C., \& Scholl, H. 2010, A\&A, 514, L4

Moorhead, A. V., \& Adams, F. C. 2005, Icarus, 178, 517

Morbidelli, A., \& Levison, H. F. 2004, AJ, 128, 2564 
Najita, J. R., Andrews, S. M., \& Muzerolle, J. 2015, MNRAS, 450, 3559

Najita, J. R., \& Kenyon, S. J. 2014, MNRAS, 445, 3315

Nesvorný, D. 2011, ApJ, 742, L22

Ostriker, E. C. 1999, ApJ, 513, 252

Rasio, F. A., \& Ford, E. B. 1996, Science, 274, 954

Raymond, S. N., Armitage, P. J., \& Gorelick, N. 2010, ApJ, 711, 772

Rephaeli, Y., \& Salpeter, E. E. 1980, ApJ, 240, 20

Rice, K. 2016, ArXiv e-prints, arXiv:1602.08390

Ruderman, M. A., \& Spiegel, E. A. 1971, ApJ, 165, 1

Takeda, H. 1988, Progress of Theoretical Physics Supplement, 96, 196

Thun, D., Kuiper, R., Schmidt, F., \& Kley, W. 2016, ArXiv e-prints, arXiv:1601.07799

Trujillo, C. A., \& Sheppard, S. S. 2014, Nature, 507, 471

Tsiganis, K., Gomes, R., Morbidelli, A., \& Levison, H. F. 2005, Nature, 435, 459

Ward, W. R. 1997, Icarus, 126, 261

Weidenschilling, S. J. 1977a, MNRAS, 180, 57

-. 1977b, Ap\&SS, 51, 153

Weidenschilling, S. J., \& Marzari, F. 1996, Nature, 384, 619

Williams, J. P., \& Cieza, L. A. 2011, ARA\&A, 49, 67

Youdin, A. N., \& Kenyon, S. J. 2013, in Planets, Stars and Stellar Systems. Volume 3: Solar and Stellar Planetary Systems, ed. T. D. Oswalt, L. M. French, \& P. Kalas, 1 
Table 1: Disk Parameters

\begin{tabular}{lcll}
\hline \multicolumn{1}{c}{ Name } & Symbol & Value or Range & Units \\
\hline radial length scale & $a_{0}$ & 1 & $\mathrm{AU}$ \\
scale height factor & $h_{0}$ & 0.05 & - \\
static disk & & & \\
surface density & $\Sigma_{0}$ & $2,10,20,50$ & $\mathrm{~g} / \mathrm{cm}^{2}$ \\
initial inner edge & $a_{\text {in }}$ & $50,100,200$ & $\mathrm{AU}$ \\
outer edge & $a_{\text {out }}$ & 1600 & $\mathrm{AU}$ \\
evolving disk & & & \\
surface density & $\Sigma_{0}$ & $50,100,200,500,1000$ & $\mathrm{~g} / \mathrm{cm}^{2}$ \\
initial inner edge & $a_{\text {in }}$ & $20,60,100,140,180$ & $\mathrm{AU}$ \\
outer edge & $a_{\text {out }}$ & 800 & $\mathrm{AU}$ \\
opening rate $\left(\dot{a}_{\text {in }}\right)$ & $\kappa_{\text {in }}$ & $20,40,60,80$ & $\mathrm{AU} / \mathrm{Myr}$ \\
decay time & $\tau$ & $2,4, \infty$ & $\mathrm{Myr}$ \\
\hline
\end{tabular}

Table 2: Planet Parameters

\begin{tabular}{lcll}
\hline \multicolumn{1}{c}{ Name } & Symbol & Value or Range & Units \\
\hline mass & $m_{\bullet}$ & $1,5,10,15,20,30,50$ & $\mathrm{M}_{\oplus}$ \\
mean density & $\rho_{\bullet}$ & 1.33 & $\mathrm{~g} / \mathrm{cm}^{3}$ \\
initial perihelion & $r_{\text {peri }}$ & 10 & $\mathrm{AU}$ \\
initial aphelion & $r_{\text {apo }}$ & $1600,2000,2400, \ldots, 3600$ & $\mathrm{AU}$ \\
inclination & $i$ & 0 & $\mathrm{rad}$ \\
\hline
\end{tabular}




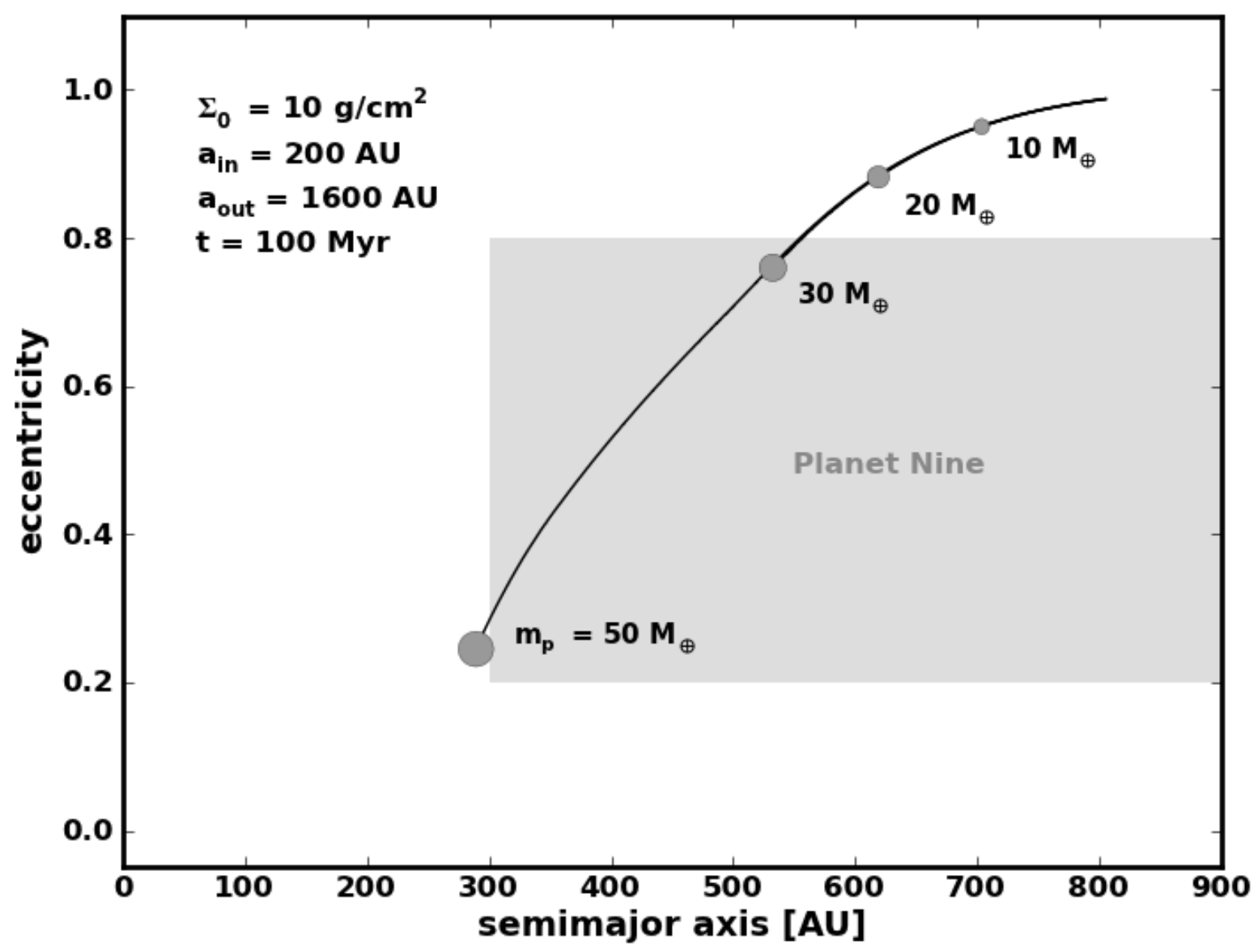

Fig. 1.- Simulations of the orbital evolution of scattered planets in a static gas disk. Solid curves show the semimajor axis $(a)$ and eccentricity $(e)$ of four planets with various masses as they evolve over a period of $100 \mathrm{Myr}$. The legend indicates the set of disk parameters. All planets start at the same high $a=800 \mathrm{AU}$ and $e=0.9875$ and evolve along a single path towards smaller values of $a$ and $e$. circular symbols show the final outcomes after $100 \mathrm{Myr}$, labeled with planet mass. More massive planets evolve faster and progress further along the path. The gray region approximates the allowed range of $a$ and $e$ from Batygin \& Brown (2016) for Planet Nine. 


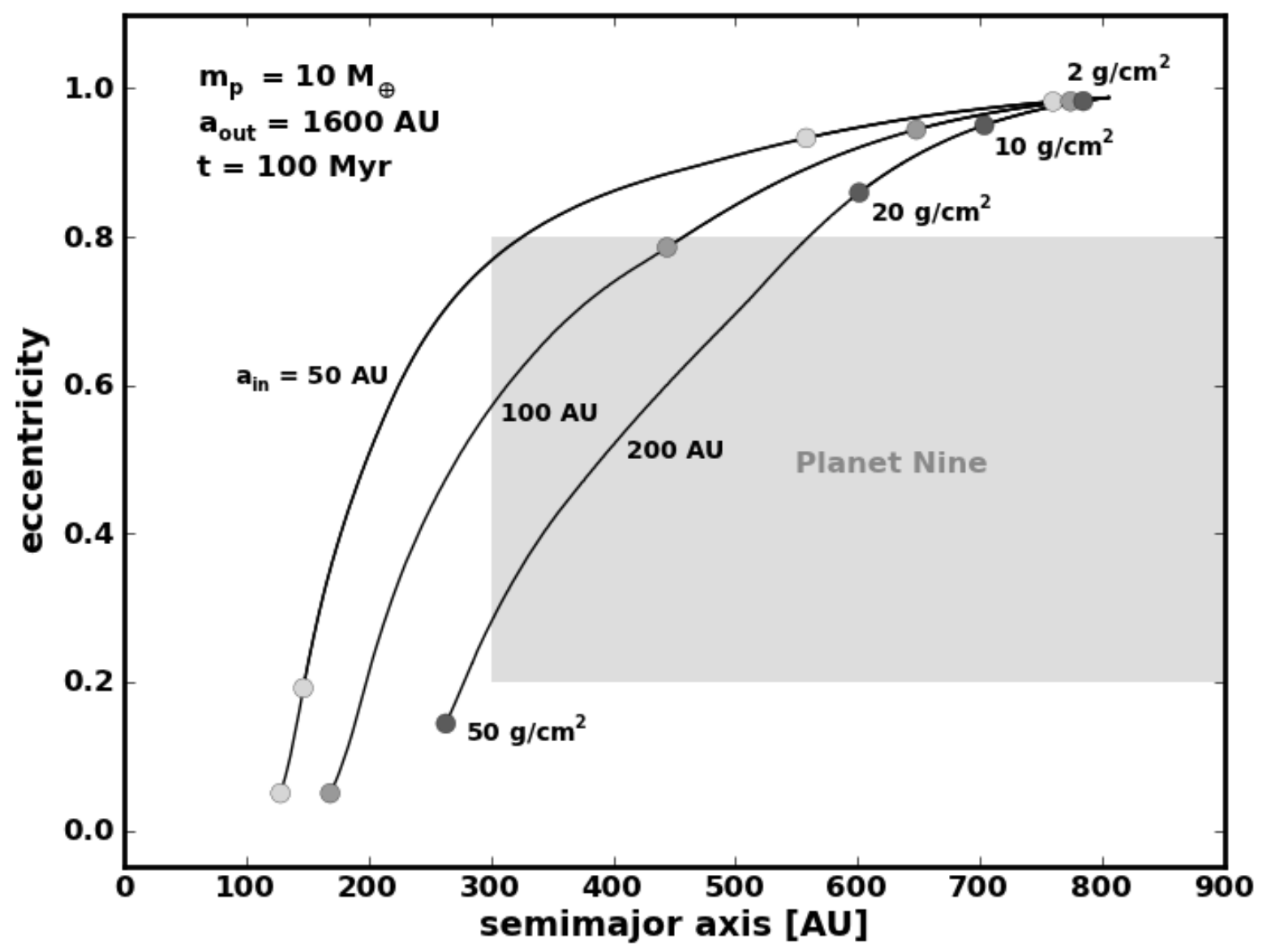

Fig. 2.- Orbital evolution of a planet in various configurations for a static disk. As in Fig. 1, planets starting with large $a$ and $e$ follow specific tracks which depend on the inner edge of the disk $\left(a_{\text {in }}\right)$. Larger inner cavities allow planets to settle at larger $a$. For a fixed planet mass of $10 \mathrm{M}_{\oplus}$, the final location in the $a-e$ plane depends on the surface density parameter $\left(\Sigma_{0}\right)$. In disks with higher surface density, orbits evolve more quickly and reach smaller $a$ and $e$. 


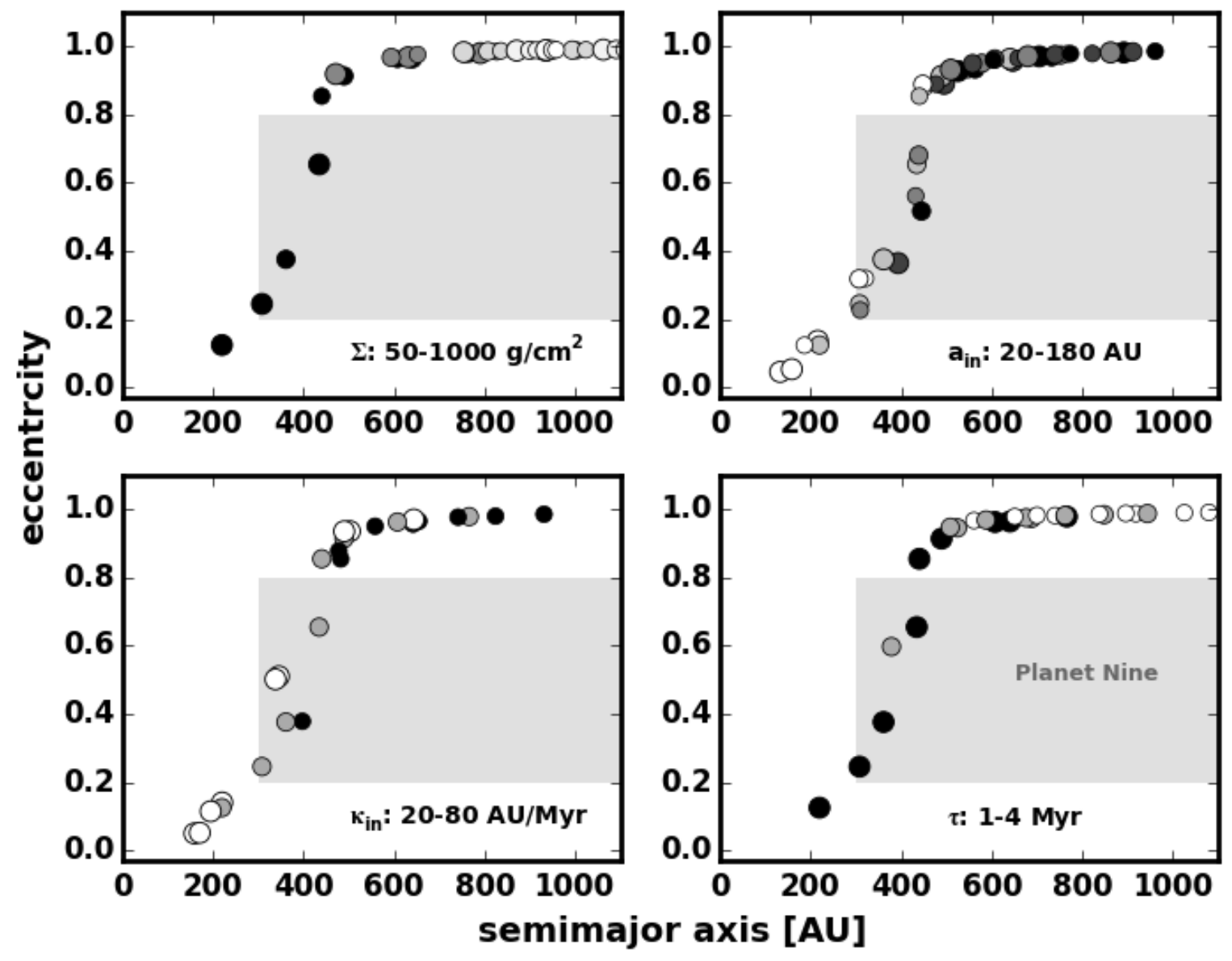

Fig. 3.- Semimajor axis and eccentricity at $10 \mathrm{Myr}$ for scattered planets with masses between $15 \mathrm{M}_{\oplus}$ and $30 \mathrm{M}_{\oplus}$ in evolving disks with baseline parameters $\left(\Sigma_{0}, a_{\mathrm{in}}, \kappa_{\mathrm{in}}, \tau\right)=\left(1000 \mathrm{~g} / \mathrm{cm}^{2}, 60 \mathrm{AU}\right.$, $40 \mathrm{AU} / \mathrm{Myr}, 4 \mathrm{Myr}$ ) and a range of initial aphelion distances (2000-2800). Each panel illustrates how outcomes change when one of these parameters is varied in the range specified in the legend. Symbol shades indicate the value of the varied parameter from white (lower values) to black (upper values). In all panels, symbol size correlates with planet mass. 


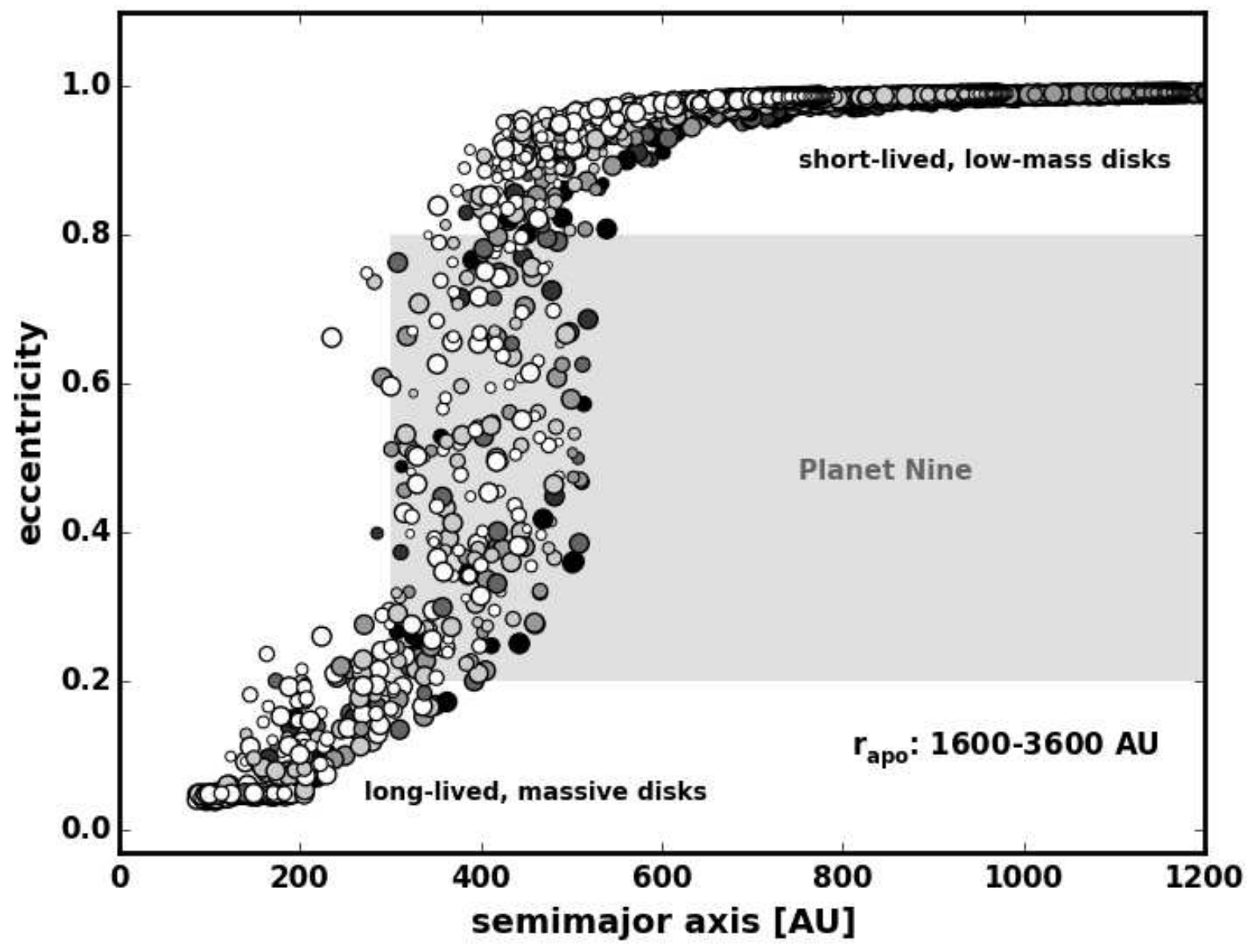

Fig. 4.- Outcomes for scattered planets at $10 \mathrm{Myr}$ in evolving disks. Symbol size correlates with planet mass; shading indicates initial aphelion distance (lightest: 1600 AU; darkest: 3600 AU). More massive planets starting at the smallest aphelion distance settle at smaller orbital distances with lower eccentricities. Variations in the initial disk configuration and the mode/time scale for disk dissipation move the final $(a, e)$ along the sequence outlined in the figure. 


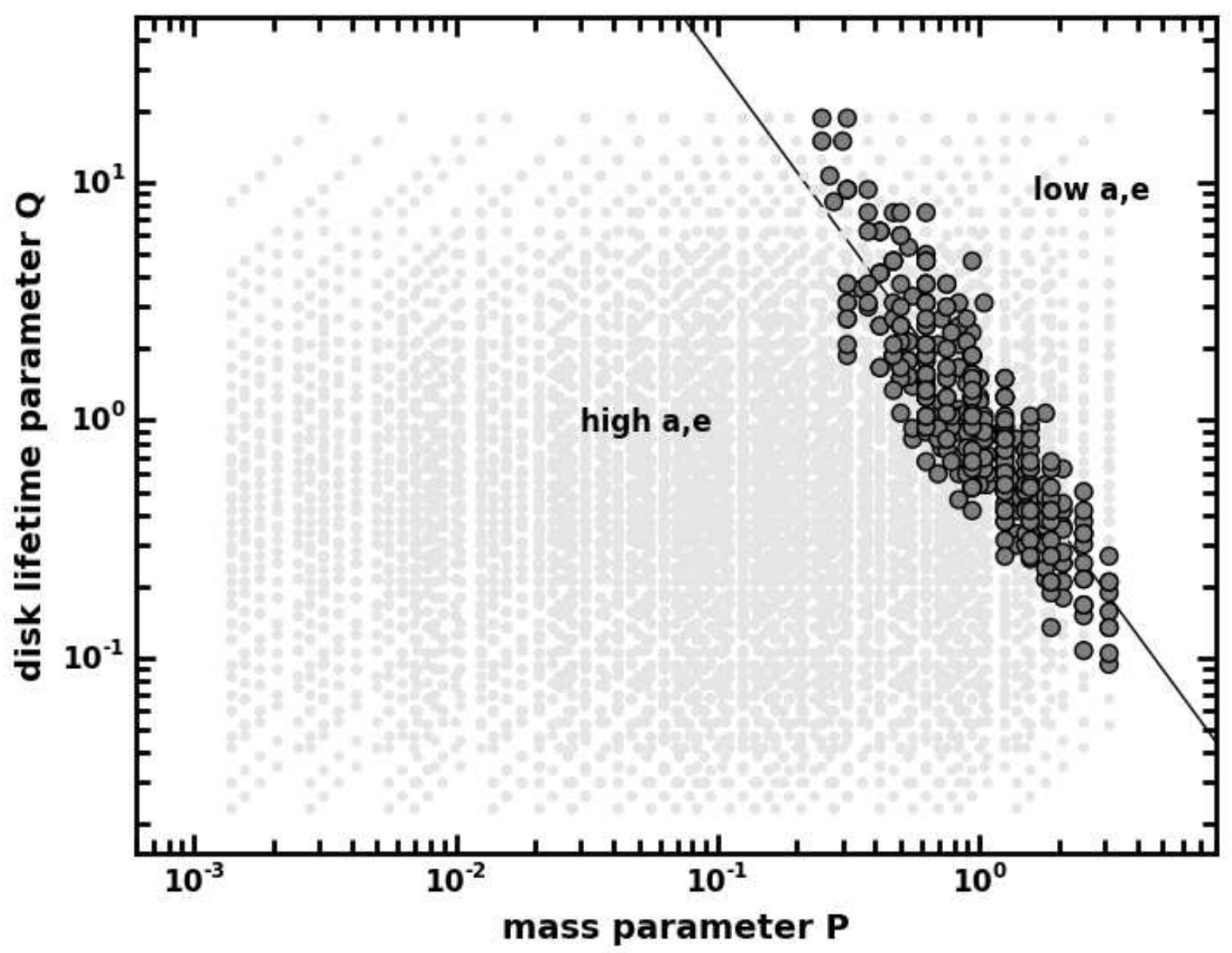

Fig. 5.- Disk lifetime and mass parameters describing outcomes for scattered planets in evolving disks. Each point in this space of mass and disk lifetime parameters ( $P$ and $Q$; see Equations (10) and (11) ) corresponds to an individual simulation with a unique set of planet and disk configurations. Dark circles with black outlines indicate successful models, which roughly match the orbital parameters of Planet Nine in Batygin \& Brown (2016) and are located in the shaded region in Figure 4. The line running through these points is from the approximation in Equation (12). The light gray points points, with the lightest shade of gray, cover the unsuccessful models where orbits are either too remote and eccentric or too close to the Sun and circular. 